\title{
Optical coherence tomography: challenge and opportunity Zhongping Chen
}

Address: Department of Biomedical Engineering, Beckman Laser Institute, University of California, Irvine, Irvine, CA 92612, USA

from Ist Scientific Meeting of the Head and Neck Optical Diagnostics Society

London, UK. 14 March 2009

Published: 28 July 2009

Head \& Neck Oncology 2009, I(Suppl I):O5 doi:I0.I I86/I758-3284-I-SI-O5

This abstract is available from: http://www.headandneckoncology.org/content/I/SI/O5

(C) 2009 Chen; licensee BioMed Central Ltd.

Optical coherence tomography (OCT) is an imaging modality based on coherence-domain optical technology. OCT takes advantage of the short coherence length of broadband light sources to perform micrometer-scale, cross-sectional imaging of biological tissue. OCT is analogous to ultrasound imaging except that it uses light rather than sound. The high spatial resolution of OCT enables noninvasive in vivo "optical biopsy" and provides immediate and localized diagnostic information. The first in vivo endoscopic OCT images in animals and humans were reported in 1997. Since then, a number of clinical applications for endoscopic OCT imaging of respiratory, urogenital, and gastrointestinal tracts have been reported by several groups. This presentation will review the principle of time domain and Fourier domain OCT and the current state-of-the-art OCT technology.

Despite the recent development of Fourier domain OCT that significantly increases imaging speed and sensitivity, the OCT system that achieves both high speed and high sensitivity simultaneously at $1.3 \mu \mathrm{m}$ is not currently available. I will describe the development of a Fourier-domainmode-lock (FDML) swept source based OCT system that can achieves high speed ( $>100 \mathrm{kHz}$ A-scan rate) and high spatial resolution $(<4 \mu \mathrm{m})$ simultaneously. In addition, the development of various miniature scanning probes that allow high-speed 3-D OCT imaging will be reported. Finally, a non-iterative digital focusing method to alleviate the compromise between lateral resolution and depth measurement range, which allows high lateral resolution over the full depth measurement range will be described. 